\title{
Los Derechos Humanos en la Política Exterior Argentina y su cobertura mediática en el período 2011-2015*
}

\author{
Antonela Busconi** \\ Jessica Marysol García*** \\ Antonella Giordano****
}

\section{Resumen}

Este trabajo en particular propone como objetivo general analizar la cobertura mediática de la temática de Derechos Humanos en la Política Exterior Argentina en el período 2011 a 2015 y se plantea como objetivos específicos identificar cuáles son los subtemas con mayor aparición vinculados a la materia en el período de estudio y observar la presencia de Memoria, Verdad y Justicia entre los subtemas asociados con Derechos Humanos.

Palabras clave: representaciones mediáticas - Cristina Fernández - Derechos Humanos - Memoria, Verdad y Justicia - Política Exterior Argentina

\section{Human Rights in the Argentine Foreign Policy and the media coverage in the period 2011 to 2015}

\begin{abstract}
This particular work proposes as a general objective to explain the media coverage of the subject of Human Rights in the Argentine Foreign Policy in the period 2011 to 2015, and so, the specific objectives are to identify which are the subtopics with greater appearance linked to the subject of Human Rights in the period of analysis and study the presence of Memory, Truth and Justice among the sub-themes associated with Human Rights.
\end{abstract}

Key words: media representations; Cristina Fernández; Human Rights; Memory, Truth and Justice; argentine foreign policy

TRABAJO RECIBIDO: 14/05/2019 TRABAJO ACEPTADO: 10/06/2019

\footnotetext{
* Este artículo se enmarca en el proyecto de investigación del Observatorio de Política Exterior Argentina (OPEA), "La Construcción de representaciones mediáticas de la política exterior argentina a través de los medios de comunicación gráfica: el Observatorio de Política Exterior Argentina 2011-2017”, el cual propone como objetivo general analizar las representaciones mediáticas construidas en torno a la Política Exterior Argentina (PEA) en medios gráficos nacionales de 2011 a 2017.

** Licenciada en Relaciones Internacionales y Doctoranda en Relaciones Internacionales, Universidad Nacional de Rosario (UNR, Argentina). Correo electrónico: antobusconi@ hotmail.com

*** Licenciada en Relaciones Internacionales, Universidad Nacional de Rosario (UNR, Argentina). Correo electrónico: jessigar1102@gmail.com

${ }^{* * * *}$ Licenciada en Relaciones Internacionales (Universidad Católica de Córdoba) y Magister en Cultura de Paz, Conflictos, Educación y Derechos Humanos. Correo electrónico: antogiordano7@gmail.com
} 


\section{Introducción}

Durante el gobierno de Cristina Fernández los Derechos Humanos se convirtieron en un eje de la política exterior. Sin embargo, ya con la llegada de Néstor Kirchner a la presidencia de la Nación se había iniciado una nueva etapa en materia de defensa de estos derechos pues en 2005 se derogaron las leyes de Obediencia Debida y Punto Final y se declararon inconstitucionales los indultos referidos a crímenes de lesa humanidad, entre otros avances dentro del tema de Memoria, Verdad y Justicia, que se produjeron en el mismo período. La presidenta Cristina Fernández continuó trabajando en el mismo sentido. La reincorporación de esta cuestión a la política doméstica tuvo proyección regional e internacional a través de la participación activa del país en los organismos internacionales (Busconi y García, 2016).

Según Corigliano (2011) las metas perseguidas por el Estado argentino en su compromiso con el régimen internacional y el sistema interno de garantía de los Derechos Humanos variaron en función de los gobiernos de turno. En el caso de los gobiernos de Néstor Kirchner y Cristina Fernández, el acento estuvo colocado en las medidas de castigo a las violaciones de estos derechos cometidas en Argentina durante la década de 1970 y sus efectos colaterales en los ámbitos regional y extrarregional. En este sentido, la centralidad discursiva del tema impulsó a estas administraciones a colocar el énfasis de la defensa de estos derechos y de la promoción democrática regional tanto en los canales bilaterales como multilaterales.

Al mismo tiempo, los medios de comunicación jugaron un rol clave en la centralidad que se le dio a la temática en la construcción de las noticias tanto en la política interna como en la política exterior durante los gobiernos kirchneristas.

Cabe destacar que en el marco disciplinar de las Relaciones Internacionales, específicamente en el campo de la política exterior, los medios de comunicación han jugado un rol clave en los cambios que se han producido en los últimos años en los modos de comunicar, pues las Cancillerías se han visto obligadas a repensar cuál es la forma más eficaz para difundir los temas de agenda (Castillo, 2015).

Teniendo en cuenta la centralidad de los Derechos Humanos tanto en la política interna como en la política exterior y, al mismo tiempo, el rol clave de los medios de comunicación en la construcción de las noticias sobre política exterior y, en particular sobre esta materia, este artículo se propone como objetivo general analizar la cobertura mediática de la temática de Derechos Humanos en la Política Exterior Argentina en el período 2011 a 2015. Para ello se plantean los siguientes objetivos específicos: identificar y evaluar los subtemas con mayor aparición vinculados con la temática en el período de estudio, y estudiar la presencia de Memoria, Verdad y Justicia entre los subtemas asociados con Derechos Humanos.

Para proceder al análisis y alcanzar los objetivos propuestos, los argumentos se focalizan en torno a las representaciones producidas por los medios de comunicación, como operadores de visibilidad y traducibilidad (Cabrelli y Rodríguez, 2013). “(...) [L]a representación mediática se articula sobre la contraposición del carácter uniforme de la expresión masiva (contexto de producción) y la diversidad de interpretaciones (contexto de recepción). Ambos componentes son el sustento concreto que permite al individuo significar los contenidos sociales ajenos, mediados e intencionados, propiciando con ello un fuerte sentido de pertenencia social" (Horta, 2013:97).

Acorde a Sary Calonge Cole, la representación mediática designa la manera en que los medios de comunicación social transmiten cierto contenido de interés colectivo, contenidos que se incorporan ulteriormente al discurso social y a la memoria de los individuos (Calonge Cole, 2006). La autora sostiene que la representación mediática posee dos dimensiones: una cognitiva y otra pragmática. La dimensión cognitiva se refiere a los trazos de orden cognitivo de la representación, esto es, a los procesos que explican la formación de representaciones sobre la realidad en las conciencias individuales en contacto con los medios. La pragmática se refiere a 
las condiciones en que los medios de comunicación social transmiten sus representaciones y al vínculo dinámico existente entre sociedad y medios.

En esta misma línea, Calonge Cole sostiene que cuando se propone investigar un objeto social a través de un medio de comunicación y cuando se quiere saber cómo es transmitido un contenido en especial por esos medios, hay dos variables que deben tomarse en cuenta: la fuente de información (¿quién habla?) y el contenido tratado (¿de qué se habla?). Ambas deben ser estudiadas y puestas en relación. El método para estudiar la representación mediática de un objeto social consiste en profundizar y relacionar el comportamiento de ambas variables al interior de un medio de comunicación social.

En la sociedad moderna, la construcción social de la realidad va de la mano de la actuación de los medios de comunicación. Esto es así, dado que gran parte del conocimiento social se adquiere a través de ellos, afirmando, cambiando o reafirmando creencias e imaginarios sociales (Sobrados León, 2011).

Parsons (2007), por su parte, afirma que los medios de comunicación incluyen o excluyen asuntos en función de lo que para ellos sea digno de ser noticia, y que sus intereses son caprichosos e inestables por lo que no todo lo que se plantea como un problema tiene la misma respuesta en todos los medios. En el caso de las políticas de la memoria es innegable que no todos los medios prestan la misma atención a las demandas que exigen verdad, justicia y reparación. En efecto, la mayoría de los medios de comunicación se desplazan en la cobertura de estos temas en función de sus intereses de grupo, de ideología o de clase social con el que se encuentren más identificados (Solís Delgadillo, 2016).

Al mismo tiempo, resulta necesario tener en cuenta que la promoción global de los Derechos Humanos puede ser una opción para muchos miembros de la comunidad internacional (Brysk, 2005:2), pues estos se han convertido en uno de los indicadores de legitimidad de los Estados en las Relaciones Internacionales (Donnelly, 1998:20).

De acuerdo a Casla Salazar, "los derechos humanos se han convertido en una idea muy poderosa que no sólo orienta las políticas internas de los gobiernos, sino que también modula sus palabras y sus acciones de puertas afuera. Los derechos humanos tienen una casilla reservada en el tablero del juego diplomático" (Casla Salazar, 2011:14).

En el caso de Argentina, los Derechos Humanos estuvieron presentes tanto en la política interna como en la política exterior, con el objetivo de renovar la imagen internacional del país y aumentar su poder blando ${ }^{1}$.

En función de lo desarrollado, se plantea como hipótesis que durante el período bajo análisis hubo coincidencia en la centralidad dada al tema Memoria, Verdad y Justicia en materia de Derechos Humanos en la Política Exterior Argentina por parte del gobierno de Cristina Fernández con la cobertura mediática de los tres periódicos de mayor tirada en el país, pero en particular con Página $/ 12^{2}$, lo cual coincide con el posicionamiento ideológico de dicho diario con las administraciones kirchneristas.

\footnotetext{
1 Joseph Nye propone una distinción entre poder duro y poder blando, como una forma de superar las limitaciones de las conceptualizaciones del poder como poder material. Específicamente, Nye caracteriza al poder blando de la siguiente manera: "refiere a la capacidad de un Estado de que otros Estados quieran secundarlo, admiren sus valores, emulen su ejemplo y pretendan alcanzar los mismos resultados que él obtuvo; consiste en la capacidad de diseñar una agenda política de forma tal, que capte las preferencias de otros actores; tiene una vinculación directa en los valores propios del Estado, que se expresan en su cultura, en las políticas que se desarrollan en el ámbito externo y en su conducta internacional" (Bartolomé, Sin Fecha).

2 Resulta necesario aclarar que en la cobertura mediática se trabaja principalmente con el periódico Página/12, pues es el medio que ha cubierto en mayor medida el tema bajo análisis en este artículo, tal como se pone de manifiesto a lo largo de este trabajo.
} 
Este trabajo se enmarca en el proyecto de investigación del Observatorio de Política Exterior Argentina (OPEA), "La Construcción de representaciones mediáticas de la Política Exterior Argentina a través de los medios de comunicación gráfica: el Observatorio de Política Exterior Argentina 2011-2017", el cual propone como objetivo general analizar las representaciones mediáticas construidas en torno a la Política Exterior Argentina en medios gráficos nacionales de 2011 a $2017^{3}$.

El primer objetivo específico de dicho proyecto es describir las características de la cobertura periodística de la política exterior en los principales medios de comunicación gráficos de alcance nacional en la Argentina y, es en este punto donde se sitúa el presente artículo.

El diseño metodológico de este trabajo es analítico descriptivo e involucra técnicas de análisis cualitativas y cuantitativas ligadas al modelo seguido por el equipo de trabajo del Proyecto de Investigación.

En lo referente al recorte temporal, se analiza el segundo gobierno de Cristina Fernández que se extiende de 2011 a 2015. Dicha selección se circunscribe al horizonte temporal propuesto por el Proyecto de Investigación en el cual se encuentra inserto este trabajo.

Se utilizan tanto fuentes primarias como secundarias. En el primer caso se trata de los comunicados de prensa de la Cancillería argentina y los informes semanales del OPEA. Mientras que en el segundo caso, se hace referencia a publicaciones de diferentes centros de investigación en la materia bajo estudio y los artículos de los periódicos.

En cuanto a las técnicas de análisis de datos, se emplean el análisis documental y el análisis de contenidos.

Finalmente, el trabajo se organiza en una introducción, un apartado donde se analiza el primer objetivo específico referido a la identificación de los subtemas con mayor aparición vinculados con la temática de Derechos Humanos; otro donde se analiza el segundo objetivo específico, la presencia del subtema Memoria, Verdad y Justicia entre los subtemas asociados con Derechos Humanos. Luego, se presentan las conclusiones.

\section{Derechos Humanos: su conceptualización en el marco de la Política Exterior Argentina}

Tal como se mencionó en la introducción, en este apartado se presentan los subtemas vinculados con la temática de Derechos Humanos con mayor aparición en los informes semanales del OPEA, pues fue uno de los temas centrales de la política tanto interna como exterior del gobierno de Cristina Fernández.

Sin embargo, antes de profundizar en el análisis de los Derechos Humanos en la política exterior del gobierno de Cristina Fernández, resulta necesario definir qué se entiende por Derechos Humanos en este trabajo. En ese sentido, y siguiendo a Herrera Flores (2003), se considera que son "el conjunto de procesos sociales, económicos, normativos, políticos y

\footnotetext{
${ }^{3}$ Luego de identificar los temas de agenda con mayor frecuencia de aparición, se determinó que la temática Derechos Humanos se encuentra en quinto lugar, junto a Relaciones Económicas Internacionales, Malvinas, Brasil, Estados Unidos y Uruguay. Una vez conocidos estos resultados se procedió a realizar en cada uno de estos subtemas el mismo trabajo que se había realizado con los temas de agenda y relaciones bilaterales. En el caso de Derechos Humanos, primero fue necesario establecer algunos criterios de unificación a fin de determinar qué temas que habían sido incorporados a los informes por separado debían incluirse en Derechos Humanos.

Una vez realizada la unificación de criterios, se procedió a la elaboración de tablas desagregadas en subtemas al interior del tema de Derechos Humanos. Se anotó la frecuencia por cada cuatrimestre y de ese modo se determinó que uno de los temas de mayor frecuencia de aparición era Memoria, Verdad y Justicia para el período 2011-2015. A partir de ello, se determinó que las noticias englobadas en Memoria, Verdad y Justicia son cubiertas en su gran mayoría por el periódico Página/12 durante el período bajo estudio.
} 
culturales que abren y consolidan -desde el 'reconocimiento', la 'transferencia de poder' y la 'mediación jurídica'- espacios de lucha por la particular concepción de la dignidad humana". En concordancia con el mismo autor se entiende que los Derechos Humanos no son algo dado y construido de una vez y para siempre sino que se trata de procesos, es decir, luchas históricas como consecuencia de las resistencias de los individuos y los colectivos ante las distintas manifestaciones del poder tanto de las burocracias públicas como privadas (Herrera Flores, 2003:27).

Entendiendo los Derechos Humanos como procesos, esta cuestión tuvo un lugar central en el discurso de los encargados de tomar decisiones de políticas públicas durante el gobierno de Cristina Fernández. En términos de práctica de la política exterior, uno de los tres pilares de la cooperación internacional al desarrollo han sido los Derechos Humanos. De esta forma, el país apuntó no solamente a ser un líder en términos discursivos, sino también a realizar aportes concretos en la defensa y promoción de los derechos fundamentales por fuera de sus fronteras. Capitalizando su experiencia en la identificación de víctimas de desaparición forzada, el país cooperó en proyectos relativos a medicina y antropología forense, bancos de perfiles genéticos y asistencia a la defensoría del pueblo en países de Latinoamérica y el Sudeste Asiático (Paraguay, El Salvador, Vietnam, Bolivia, Colombia, Ecuador y Tailandia) (Pauselli, 2014).

No obstante, la administración de Fernández fue blanco de críticas por su política exterior en la materia en diversos ámbitos. Estas críticas provinieron generalmente de sectores opositores, puesto que desde sectores oficialistas, se afirmaba que ningún gobierno argentino había hecho tanto por los Derechos Humanos como el de Fernández (Pauselli, 2014).

Así también, Argentina posee unos de los niveles de firma y ratificación de los tratados internacionales de Derechos Humanos más alto del mundo. Fue uno de los principales promotores de la Convención Internacional para la Protección de todas las Personas contra las Desapariciones Forzadas, de la que actualmente son parte 42 Estados. En el ámbito regional, se destaca la participación activa de Argentina de la redacción de la Resolución 2662, denominada "El derecho a la verdad", aprobada por la Asamblea General de la Organización de Estados Americanos (OEA), a partir de la cual se reconoce la importancia de respetar y garantizar el derecho a la verdad para contribuir de esta manera a terminar con la impunidad y promover y proteger los Derechos Humanos (Busso, 2016).

Asimismo, durante este período el Estado argentino tuvo un rol activo en organismos internacionales. Por ejemplo, dentro del Consejo de Derechos Humanos de Naciones Unidas, el país fue el promotor de varias iniciativas como la adopción del derecho a la verdad, el uso de la ciencia forense para la investigación de violaciones de Derechos Humanos, y la creación del Relator Especial para la promoción de la verdad, la justicia, la reparación y las garantías norepetición.

Es en este marco que cobra importancia el análisis de la aparición de la representación mediática del tema pues, en general, tiene una frecuencia de aparición que supera el 20 por ciento en todos los cuatrimestres en el período 2011-2015, alcanzando más del 83 por ciento en el tercer cuatrimestre de 2014 (Ilustración 1. Gráfico de elaboración propia).

A los fines de este trabajo de investigación, se agrupan dentro de Derechos Humanos una variedad de subtemas que incluyen todas aquellas noticias relacionadas con la defensa y promoción de los derechos humanos, en el ámbito doméstico e internacional, durante el período bajo estudio.

Teniendo en cuenta lo anterior, como se puede observar en la segunda ilustración, dos de los tres temas que aparecen con mayor frecuencia, como son Memoria, Verdad y Justicia y Libertad de Prensa y Expresión, dan cuenta de la relevancia que tuvo la temática durante el gobierno de Cristina Fernández y su representación en los medios. Además, como se mencionó anteriormente, los dos temas muestran la intención del gobierno argentino de convertirse en un 
referente de la defensa de los estos derechos en el exterior por un lado, y las críticas desde otros sectores sobre restricciones a la libertad de expresión por el otro.

Cabe destacar, no obstante, que el tema que con mayor frecuencia aparece es el primero, seguido en algunos cuatrimestres por Cascos Blancos y en otros Libertad de Prensa y Expresión y Jurisdicción Universal, a excepción del segundo cuatrimestre de 2011 donde el subtema con más frecuencia de aparición es la Comisión Interamericana de Derechos Humanos (CIDH).

La aparición constante de Memoria, Verdad y Justicia, tiene directa relación con el posicionamiento que los gobiernos kirchneristas y, especialmente, el de Cristina Fernández, quisieron darle al tema. Como sostiene Bietti, se cambió el discurso previo oficial sobre el pasado. El nuevo posicionamiento de estos gobiernos ha sido recalcado desde la legitimación del discurso de las víctimas (Bietti, 2009). Particularmente, durante los gobiernos de Cristina, se reabrieron muchas causas penales y se condenaron violaciones de los Derechos Humanos que fueron cometidas durante el régimen militar.

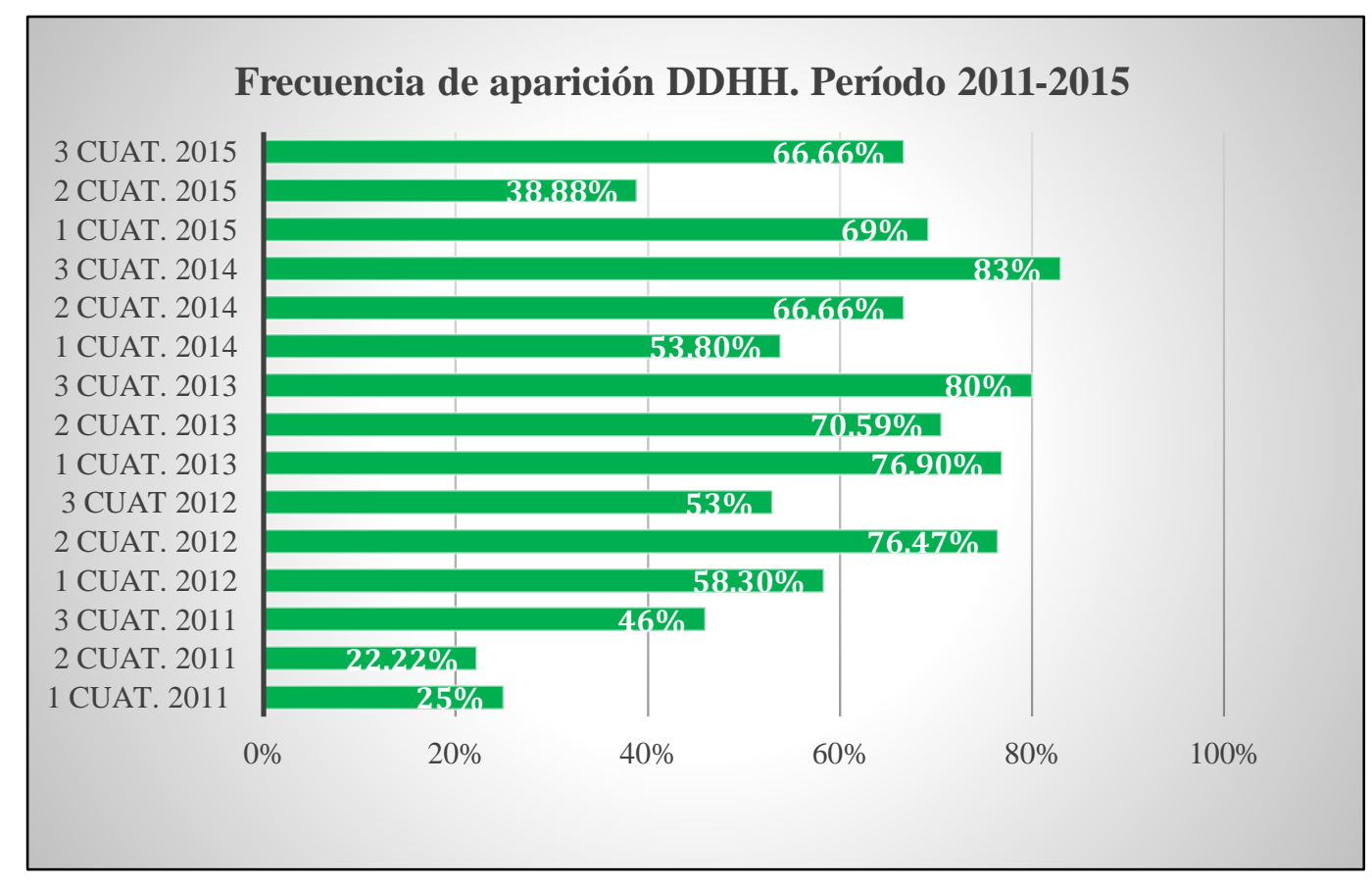

Ilustración 1. Gráfico de elaboración propia

En cambio, Libertad de Prensa y Expresión es un tema cuya aparición estuvo ligada a las presuntas denuncias de falta de garantías a las libertades de prensa y expresión por parte del Estado argentino, esgrimidas por determinados medios de comunicación y por los informes presentados por distintas organizaciones en cuanto a la falta de cumplimiento de estas libertades. Asimismo, cabe destacar que en este caso su aparición en organismos u organizaciones no gubernamentales internacionales no estuvo en relación con una política del gobierno de Cristina Fernández, sino que fue consecuencia de las demandas de algunos de los medios de comunicación argentinos ante su desacuerdo con la sanción de la denominada Ley de Medios en 2009. En este sentido, la sanción de dicha ley fue presentada por el gobierno argentino como un recurso para lograr equidad y pluralidad en el acceso como en la producción de la información, alcanzando de ese modo a terminar con los monopolios informativos, en los cuales se incluía el Grupo Clarín, dueño de uno de los medios anteriormente mencionados. 
En cuanto a la aparición de Cascos Blancos, la misma tiene relación con la estructuración de la Comisión Cascos Blancos durante la gestión de Cristina Fernández como una herramienta de la Política Exterior Argentina a fin de contribuir a la inserción del país en el mundo, tanto a nivel regional como multilateral (Cascos Blancos, 2014: 143).

Mientras que en el caso del tema Jurisdicción Universal, las noticias relevadas se refieren a crímenes cometidos por el franquismo en España que ante la imposibilidad de juzgamiento en ese país se han entablado acciones en Argentina. Si bien no hay relación directa entre los crímenes cometidos por la última dictadura en Argentina y los cometidos por el régimen franquista en España, sí hay coincidencia en la demanda constante de justicia por parte de las víctimas de ambos regímenes.

La aparición de la CIDH, como órgano principal y autónomo de la Organización de los Estados Americanos (OEA) que tiene el mandato de promover la observancia y la defensa de los Derechos Humanos en la región, tampoco sorprende. La marcada presencia de noticias sobre la temática relativa a Memoria, Verdad y Justicia, de la cual el país es promotor desde hace décadas en la región, como de las críticas de la CIDH en materia de protección de la libertad de expresión y de prensa, están en coincidencia con dos de los temas que tuvieron una mayor preponderancia en el período bajo análisis.

A diferencia de los temas anteriores, la aparición del subtema Memoria, Verdad y Justicia se condice con las medidas de política exterior destinadas a la promoción y defensa de los estos derechos implementadas por Cristina Fernández durante su período de gestión. En este sentido hubo coincidencia entre la decisión tomada por el gobierno argentino en materia de política exterior, con la frecuencia de aparición del tema en los tres periódicos de mayor tirada en Argentina. No obstante, resulta necesario tener en cuenta que ni la frecuencia ni la representación sobre el tema es la misma en estos tres medios.

Cabe recordar al respecto que, durante la última dictadura militar de Argentina, parte de la prensa participó activamente del golpe. Mientras una parte del periodismo resistió, otra parte actuó sistemáticamente para legitimar el crimen: la desaparición forzada de personas, las torturas, los robos de bebés, el saqueo del país a través de un plan económico que dejaría por décadas fuera de la vida vivible a millones de argentinos (Saintout, 2013).

En este contexto puede vislumbrarse el porqué de la estrecha vinculación entre la presidencia de Cristina Fernández y el periódico Página/12. Los medios toman posturas y representan intereses e ideologías. Es así que se los suele entender cercanos a posturas políticas o sociales diversas, y ello se ve representado en la cobertura mediática que realizan. En este sentido es destacable que la mayoría de las noticias referidas a esta materia y, en particular, a Memoria, Verdad y Justicia aparecen en el diario Página/12.

Desde sus inicios Página/12 se caracterizó por ofrecer "costados distintos", brindar información sobre grupos minoritarios e incluir audacia en sus publicaciones. El diario nació en 1987 con estilo pluralista y progresista y con un manifiesto compromiso con la democracia. Además, el medio intentó continuar con la tarea del Diario La Opinión, censurado en los años 70 por la dictadura militar ${ }^{4}$.

Página/12 buscó informar sobre grupos minoritarios e influyentes en la sociedad -gays, lesbianas, feministas, ecologistas, psicoanalistas y militantes de los Derechos Humanos-; con buen criterio recuperó la importancia de dos secciones poco a poco relegadas en otros medios, Educación y Universitarias-, así como tomó entre sus banderas permanentes el análisis y marcha

\footnotetext{
${ }^{4}$ Página/12 en su historia argumenta que "este país necesitaba un medio pluralista con un único compromiso con la democracia y los derechos humanos. Que sirviera para informar con independencia". Asimismo, el diario define su estilo de informar por "la trasgresión, la defensa de los derechos humanos y la ironía, lo cual (...) provocó una adhesión permanente de parte de sus lectores" (Página/12Institucional). Disponible en: https://www.pagina12.com.ar/usuarios/institucional.php. Consulta: $20 \mathrm{de}$ noviembre de 2018
} 
de asuntos como la justicia, los militares (que venía junto con la revisión del pasado reciente) y la corrupción en el mundo político (Ulanovski, 1997).

El interés por los Derechos Humanos y el repudio a las prácticas militares vincularon a Página/12 con la gestión de Cristina Fernández, quien convirtió ambos temas en dos de sus principales estrategias políticas. Asimismo, los principales periodistas de Página/12 declararon públicamente su militancia política y su adhesión al partido kirchnerista.

En concordancia con esto, el diario publicó, en su edición del 30 de mayo de 2012, declaraciones de Cristina Fernández. La ex mandataria calificó al medio como "una contraseña de identidad" y destacó que "25 años de Página/12 son 25 años de un país que reclamaba cosas, que hoy estamos haciendo también colectivamente". En esta línea, agregó "muchas gracias, primero por haber sostenido, durante estos 25 años contra viento y marea Página/12, es un mérito que todos tenemos que agradecer (...)"

Con anterioridad a la llegada de Cristina Fernández a la presidencia, Página/12 “(...) construyó un posicionamiento próximo al Gobierno en términos político-ideológicos. En tanto institución política, el periódico se mostró interpelado directamente por los anuncios que compusieron la propuesta de recuperación del primer kirchnerismo y, al interpretar la actualidad, activó sus propios marcos ideológicos que lo ubicaron cerca de la política oficial" (de Diego, 2014:330).

\section{2 - Memoria, Verdad y Justicia}

En este apartado se analiza el segundo objetivo específico, la presencia Memoria, Verdad y Justicia entre los subtemas asociados con Derechos Humanos, pues como se mencionó en el apartado anterior y tal como puede observarse en la Ilustración 2, es el subtema que aparece con mayor frecuencia en el período bajo análisis.

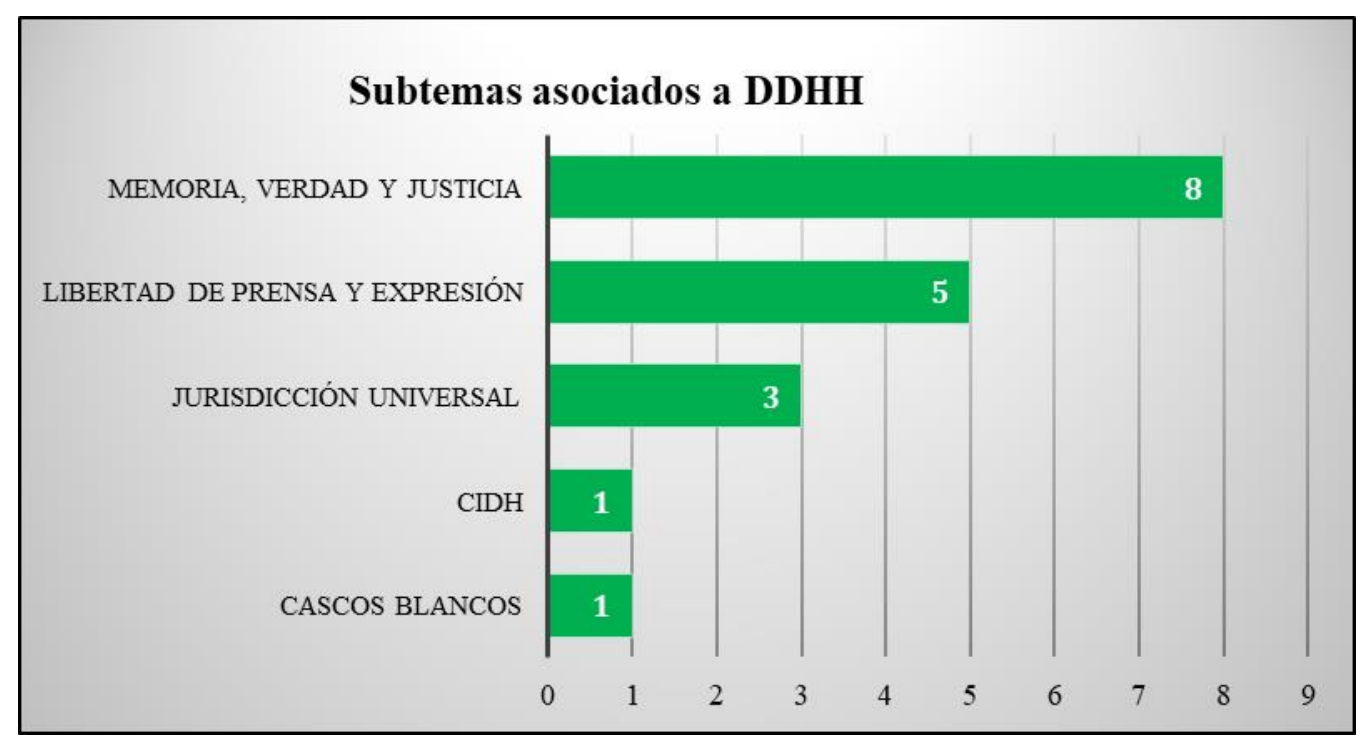

Ilustración 2. Gráfico de elaboración propia

\footnotetext{
5 "Página es una contraseña de identidad", Página/12, 30 de mayo de 2012. Disponible en: https://www.pagina12.com.ar/diario/especiales/18-195322-2012-05-31.html. Consulta: 20 de noviembre de 2018
} 
No obstante, antes de avanzar en el análisis de la aparición de este subtema en los informes del OPEA del período 2011-2015, resulta necesario aclarar qué se entiende por Memoria, Verdad y Justicia en el marco de este trabajo. Teniendo en cuenta que existen diversas miradas sobre este tema al interior de la misma sociedad argentina y que el análisis se concentra en la política exterior y no en la política interna, en este trabajo se incluyen bajo el eje Memoria, Verdad y Justicia los juicios a los responsables de los crímenes de lesa humanidad cometidos durante la última dictadura militar, la búsqueda de los niños y niñas expropiados y de las personas desaparecidas durante ese período, así como las acciones llevadas a cabo por los diferentes movimientos de Derechos Humanos para encontrar a dichas personas y reivindicar la necesidad de juzgar a los responsables, y la actividad desplegada por el gobierno argentino a nivel internacional en la búsqueda de personas desaparecidas no sólo en Argentina sino también en el exterior.

La gestión de Cristina Fernández asumió como política de Estado la condena a las violaciones de estos derechos cometidas durante la última dictadura militar, enarbolando la cuestión de la memoria como una bandera a seguir, apoyando en este sentido las reivindicaciones históricas de los movimientos de defensa de los Derechos Humanos como la Asamblea Permanente por los Derechos Humanos (APDH) y Madres y Abuelas de Plaza de Mayo, entre otras (Moreira, Barbosa, 2010).

Aquí cabe destacar el hincapié que hizo la administración de Fernández por juzgar a los represores de la última dictadura y sus cómplices en las esferas civil y religiosa, lo cual llevó a solicitar las extradiciones de dichos represores ante gobiernos del exterior.

De acuerdo al Centro de Estudios Legales y Sociales (CELS), durante la década que se extiende de 2001 a 2011, se consolida el juzgamiento de los responsables de dichos crímenes, luego de pasados más de treinta años de cometidos los mismos. Esta organización también reconoce que ello es el resultado de la lucha política y del diseño de innovadoras estrategias sociales y jurídicas por parte del movimiento de Derechos Humanos en Argentina y esta consolidación fue posible gracias a un contexto interno y externo favorable al juzgamiento (Balardini, 2012).

Siguiendo este contexto histórico, el número de apariciones de las noticias referidas a la violación de los Derechos Humanos durante la última dictadura militar, englobadas dentro de la categoría Memoria, Verdad y Justicia, tiene su razón de ser ya que fue uno de los estandartes de defensa de este gobierno, tanto a nivel doméstico como internacional. De igual modo, el hecho de que Página/12 sea el principal medio gráfico de comunicación en el que estas noticias han sido publicadas, también tiene su explicación en el vínculo político-ideológico con el gobierno de Cristina Fernández.

El discurso oficial, con el gobierno de Cristina, se ha apropiado de demandas históricas, dándole forma, y nucleando organizaciones sociales y de Derechos Humanos, implantándose en su terreno. Un espacio en el que, hasta entonces, era solo de la sociedad civil (Bietti, 2009). Esta relación de apropiación de la temática por el gobierno de Cristina Fernández también es destacada por Glanc (2008).

La memoria como ejercicio colectivo y reciente, particularmente relativo a la dictadura militar de 1976 a 1983, se intenta recobrar como una representación discursiva de lucha, como parte de un ejercicio colectivo mayor que incluye manifestaciones varias y con variados actores. Es una memoria que, dado que tuvo que ver con actos del Estado, entra en relación directa con la historia reciente y la cosa "pública" (Torres, sin fecha).

En este sentido, cabe resaltar que el gobierno ha tomado una clara postura en relación al tema que nos atañe, Memoria, Verdad y Justicia, desde la perspectiva de las víctimas, los militantes, y los enemigos en un modelo que explica las prácticas de la dictadura a la que refiere. Ello ha intentado ser construido como política de Estado por el gobierno de Cristina Fernández, tanto a través de los medios de comunicación afines al gobierno, como por el trabajo 
de organismos de Derechos Humanos, a través de una inversión particular del Estado con el objetivo de estimular y favorecer el relato de esta memoria colectiva y la legitimación de un modelo de sociedad en lucha contra ello (Torres, sin fecha).

Bravo (2012), destaca el hecho de que estos esfuerzos del gobierno de Cristina Fernández, han logrado implantar un acuerdo social en torno a la necesariedad de una lucha por la justicia y el repudio a los gobiernos de facto que azotaron al país.

La relación particular con algunos organismos de Derechos Humanos fue recalcada por varios espacios. La recepción de Madres de Plaza de Mayo, la presencia de estos organismos en actos públicos, la elección en cargos públicos de personas afines a los espacios de lucha por los Derechos Humanos, todo ello dio cuenta de esta nueva relación del gobierno con la temática que aquí nos ocupa.

Cabe destacar que, previamente, con la llegada de Néstor Kirchner al poder, se produce un cambio en el marco de acción acerca de la política y el Estado por parte de Madres de Plaza de Mayo, en gran medida, gracias a la aparición de nuevas oportunidades políticas. Esto combinado con la puesta en marcha de una dimensión afectiva en torno a la figura de Néstor Kirchner, conduce a Las Madres a tomar como propio el proyecto político inaugurado en 2003 (Andriotti Romanin, 2012a).

En el mismo sentido, Abuelas de Plaza de Mayo es una de las organizaciones que aparece en varios de los informes del OPEA en el período bajo análisis, en particular en acciones llevadas a cabo fuera del país para difundir el Derecho a la Identidad. Cabe destacar, además, la creación de la Casa de la Identidad, en 2014, en el Espacio Memoria y Derechos Humanos (ex ESMA).

A ello se le puede sumar la revaloración que los mismos espacios dedicados a la Memoria en Argentina le han otorgado a la labor de la ex presidenta. En el marco de la inauguración de algunos espacios en la ex ESMA, se le entregó en 2015 el premio "Hacedores de la Memoria" por su compromiso con los Derechos Humanos ${ }^{6}$.

En una publicación de diciembre de 2015 en la Plataforma de Cristina Fernández en su sitio personal web (www.cfkargentina.com) se mencionan todas las políticas relativas al tema de Memoria, Verdad y Justicia en toda la gestión (2003-2015) como uno de los grandes ejes de política ${ }^{7}$.

Tanto Madres como Abuelas de Plaza de Mayo se constituyeron, de acuerdo a Andriotti Romanin (2012b), en "los aliados" de los gobiernos kirchneristas al interior de los movimientos de Derechos Humanos de Argentina. "Un elemento que los define es el apoyo activo a las políticas del gobierno más allá de las estrictamente referidas a derechos humanos y su identificación política con el mismo" (Andriotti Romanin, 2012b:61). No obstante, hubo otros organismos que si bien no se constituyeron en aliados, sí comparten en general una mirada positiva acerca de las políticas de derechos humanos impulsadas por estos gobiernos. Entre ellos, Andriotti Romanin incluye a Madres de Plaza de Mayo - línea Fundadora-, la Asamblea Permanente para los Derechos Humanos (APDH) y el CELS, a los cuales denomina "colaboradores" (Andriotti Romanin, 2012b:61).

En este sentido, el protagonismo que asumen estos organismos constituidos en su mayoría durante la última dictadura militar va de la mano del protagonismo otorgado a la

\footnotetext{
6 La Presidenta Cristina Fernández recibió el premio "Hacedores de la Memoria", Espacio Memoria y Derechos Humanos, 3 de diciembre de 2015. Disponible en: http://www.espaciomemoria.ar/2018/noticia.php?not_ID=724\&barra=noticias\&titulo=noticia. Consulta: 20 de noviembre de 2018

${ }^{7}$ Información extraída de https://www.cfkargentina.com/memoria-verdad-y-justicia/. Consulta: 20 de noviembre de 2018
} 
temática Memoria, Verdad y Justicia durante las administraciones de Néstor Kirchner y Cristina Fernández.

Asimismo, tal como resalta Bietti (2009), en consonancia con lo dicho anteriormente, Página/12 desde 2004 tiene una alta presencia en cuanto a la publicación de noticias relacionadas a la desaparición de personas durante la última dictadura militar, así como a la reapertura de procesos judiciales, entre otras cuestiones.

Sobre la relación del diario con el gobierno nacional, es importante señalar que es reconocida su posición cercana al gobierno de Cristina Fernández, siendo cuestionado como "oficialista". Torres manifiesta esta relación, al plantear que:

Horacio Verbitsky en una entrevista responde sobre estos cuestionamientos desde su rol de referente periodístico y de asesor del CELS sobre Derechos Humanos, que no se volvió Página/12 oficialista sino que sobre los puntos cruciales de esta agenda el diario y el presidente electo coincidían. "Entonces él me dice: "Mi gobierno en este tema se a basar en tres puntos: memoria, verdad y justicia". Le digo: "Bueno, veamos. Si es así, nos vamos a llevar bien, si no no" (sobre el primer encuentro con Kirchner, el ejemplo vale para aclarar que la relación con el poder se condicionaba a temas que Página consideraba no negociables) (Torres, Sin Fecha:11).

Es notoria la relación de la dimensión pragmática de las representaciones mediáticas en la aparición del tema Memoria, Verdad y Justicia en Página/12, con mayor frecuencia que en los otros medios citados. Ello, dado que concuerda con expresiones periodísticas dedicadas a legitimar las políticas de gobierno de Cristina Fernández y a reforzar una identidad política, en concordancia con lo que algunos autores denominan "periodismo militante" (Fernández, 2013). Es así, que queda explicitado el vínculo que esta dimensión tiene respecto de la relación sociedad y medio.

Teniendo en cuenta que los medios de comunicación incluyen o excluyen asuntos en función de sus intereses (Parsons, 2007), en el caso de Página/12 la mayor aparición de los temas referidos a Derechos Humanos, en general, y Memoria, Verdad y Justicia, en particular, visibiliza intereses de grupo o de ideología con que el medio gráfico se siente más identificado.

De acuerdo a de Diego (2014), Página/12 reconoció en el Gobierno de Néstor Kirchner:

(...) una impronta setentista, en la cual leyó la puesta en valor de la oportunidad histórica de un colectivo político que había sido ladeado históricamente de los espacios de poder, y que ahora asumía la gestión nacional, revalorizando algunas luchas y valores del pasado. Se activaron así marcos ideológicos que históricamente habían determinado la producción discursiva del periódico (el recuerdo positivo de la militancia de los '70, los debates en torno a la justicia y los derechos humanos, la memoria y el pedido de castigo respecto de los crímenes de la última dictadura, entre muchos otros) (de Diego, 2014:337).

En este sentido, la vinculación político-ideológica entre Página/12 y el gobierno progresista de Cristina Fernández responde a que el diario representó un espacio político de izquierda que iba desde radicales progresistas hasta independientes de izquierda de lo más variados. No había un grupo político organizado que representara estos valores (defensa de los Derechos Humanos, justicia social, entre otros), con lo cual no tardó en hacerse un lugar entre los principales periódicos de tirada nacional (Zunino, 2009).

De acuerdo con esto, en un artículo del 26 de noviembre de 2013, el periódico destaca los avances de los últimos 30 años en la consolidación de leyes que reconocen como nuevos sujetos de derecho a personas antes marginadas “(...) los hijos de desaparecidos pasaron a ser sujetos de derecho con los juicios de DD.HH. y las políticas de la memoria que les devolvieron su identidad, les devolvieron un sentido a la justicia, a la jurisdicción, a los testimonios" (Croxatto, 2013).

Esto nos permite inferir la importancia que Página/12 confiere a las políticas de Memoria, Verdad y Justicia que caracterizó la política interna y exterior de Fernández. 
Esta aproximación entre medio y gobierno se manifestó a lo largo de todo el mandato de Fernández, incluido su último año de gestión. Por ejemplo, en enero de 2015, uno de sus columnistas defiende las políticas de Memoria, Verdad y Justicia que la administración kirchnerista implementó frente a los argumentos que este semanario consideró que Mauricio Macri, por entonces jefe de gobierno de la ciudad de Buenos Aires, esgrimió para desprestigiar estos logros:

Tres han sido, hasta ahora, las argumentaciones del macrismo en esta ofensiva que avanza por los márgenes: condenar los curros que se habrían cometido al amparo de las políticas de derechos humanos, haciendo referencia al caso de Sergio Schoklender; señalar (...) que una atención excesiva por los temas del pasado podía llevar a desatender los derechos humanos del presente, y finalmente que esas políticas se estaban llevando a cabo con un ánimo de venganza que tendría más peso que el afán de justicia (Jozami, 2015).

En sintonía con lo anterior, en otro artículo publicado por Página/12 en octubre de 2015, se destaca la agenda que entre el 2013 y 2015 Argentina promovió como miembro del Consejo de Derechos Humanos. Se pone de relieve la "(...) defensa integral de los derechos humanos y la no discriminación basada en el interés de las víctimas, sin importar cuál sea el gobierno responsable de las violaciones" y las "(...) acciones en favor del reconocimiento internacional del derecho a la verdad, a la justicia y contra la impunidad en los casos de violaciones sistemáticas y flagrantes a los derechos humanos". En esta editorial se menciona también el esfuerzo de Argentina, junto a Suiza y Marruecos, "en el reconocimiento internacional del derecho a la verdad y a la justicia y contra la impunidad como bases indispensables y garantía de los procesos de transición a la democracia luego de períodos de dictadura o guerras civiles". Así también, se recalca la tarea del país junto a Francia en la difusión en favor de la ratificación de la Convención Internacional contra la Desaparición Forzada de Personas, de apoyo al Grupo de Trabajo sobre Desapariciones Forzadas y otras acciones de denuncia, contando en tres oportunidades con la participación en el plenario y en eventos paralelos de Estela de Carlotto, presidenta de Abuelas de Plaza de Mayo, quien aportó el testimonio de su lucha (D'Alotto, 2015).

Como queda evidenciado, Página/12 fue el principal medio de comunicación en que se publicaron las políticas referidas a la cuestión Memoria, Verdad y Justicia, durante la gestión de Cristina Fernández.

De igual forma, la concordancia ideológica de los periodistas con el gobierno se manifiesta en las opiniones vertidas en el mismo. En la sección Opinión, el periodista Mario Wainfeld, meses previos a las elecciones presidenciales de 2015, destaca las políticas y medidas adoptadas durante la gestión de Cristina Fernández tanto en materia laboral y social como en el ámbito de los Derechos Humanos:

(...) Jubilaciones cuasi universales, con aumentos semestrales estipulados por ley. La Asignación Universal por Hijo (AUH), innovación de la etapa que se actualiza de igual modo. Las convenciones colectivas libres y en alza. El Consejo del Salario mínimo vital y móvil. La paritaria nacional docente. Los programas Progresar y ProCrear. Los nuevos regímenes para empleadas de casas particulares y trabajadores rurales. No es poco, ni se enumeran todos. En otro carril, se legisló el matrimonio igualitario y normas ampliatorias de derechos de minorías y de género (Wainfeld, 2015).

Por consiguiente, Página/12 se caracterizó por tener una matriz informativa más proclive a una mayor diversidad de fuentes y puntos de vista y manifestó una clara intencionalidad editorial favorable, tanto a las posiciones del gobierno de Fernández como a contrarrestar los contenidos de carácter cuestionador de la institucionalidad política y social del país (Zunino, 2009). Se puede concluir que en los últimos tiempos, Página/12 se caracterizó por desarrollar un discurso afín a las políticas más importantes del gobierno nacional, entre las que se destacan las referidas a Memoria, Verdad y Justicia (Koziner y Zunino, 2013). 


\section{Conclusión}

Tal como se mencionó en la introducción, este trabajo se enmarca en el primer objetivo del proyecto de investigación del OPEA, en el cual se plantea describir las características de la cobertura periodística de la política exterior en los principales medios de comunicación gráficos de alcance nacional en la Argentina. En ese sentido, y en el caso particular de la cobertura del tema Derechos Humanos, ha podido observarse que la construcción social va de la mano de la presencia mediática. En este caso los medios notoriamente han tenido participación en la construcción social de la temática en general de Derechos Humanos, y en particular de Memoria, Verdad y Justicia.

Por un lado, el gobierno ha seleccionado la temática de Derechos Humanos y en particular Memoria, Verdad y Justicia como una política de Estado, y como un frente de lucha en su manifestación social. Como se puso de manifiesto a lo largo de este trabajo, este tema no sólo fue central en la política interna del gobierno de Cristina Fernández sino también en materia de política exterior, lo cual también quedó explicitado en la cobertura mediática y, especialmente en el caso de Página/12.

El medio de comunicación Página/12, ha hecho suyo el subtema a través de la RM del mismo al largo del período 2011-2015. Esto se manifestó en la presencia mayoritaria del medio cubriendo noticias relativas a Memoria, Verdad y Justicia en el período analizado, y en la manifiesta vinculación del medio con la temática general de Derechos Humanos, explicativa de la anterior relación.

Entonces, es posible sostener que en el período bajo análisis existió una confluencia en la importancia que el tema Memoria, Verdad y Justicia tuvo dentro del pilar de Derechos Humanos de la Política Exterior Argentina por parte de la administración de Cristina Fernández con la representación mediática de los tres periódicos de mayor tirada del país sobre esta cuestión, pero en especial con Página/12, lo cual coincide con el posicionamiento ideológico de este diario con las administraciones kirchneristas.

\section{Bibliografía}

ANDRIOTTI ROMANIN, E. (2012a). De la resistencia a la integración. Las transformaciones de la Asociación Madres de Plaza de Mayo en la "era Kirchner", en Estudios Políticos, $N^{\circ}$ 41, Medellín, Julio/Diciembre. $\quad$ Disponible en: $\quad$ http://www.scielo.org.co/scielo.php?pid=S012151672012000200003\&script=sci_arttext\&tlng=pt. Consulta: 11 de agosto de 2018

ANDRIOTTI ROMANIN, E. (2012b). De la confrontación a la cooperación. Los cambios en las estrategias y marcos interpretativos del Movimiento de derechos humanos de Argentina frente al "kirchnerismo" (2003-2011), en TEJERINA, B. y PERUGORRÍA, I. (editores), Global Movements, National Grievances. Mobilizing for "Real Democracy" and Social Justice. Universidad del País Vasco, $\quad$ pp. 53-68. Disponible https://www.researchgate.net/profile/Begonya Enguix/publication/305565246_Cuerpos_Camisetas e_Identidades_como_Estrategias_de_Protesta/links/57938a8a08aec89db7924bd6/CuerposCamisetas-e-Identidades-como-Estrategias-de-Protesta.pdf\#page=53 Consulta: 11 de agosto de 2018

BALARDINI, L. (2012). A diez años de la nulidad de las leyes de impunidad. La consolidación del proceso de justicia por crímenes de lesa humanidad en la Argentina. Los nuevos debates y los problemas aún sin resolver. Derechos humanos en Argentina. Informe 2012, Capítulo I, CELS. Disponible en: https://www.cels.org.ar/web/publicaciones/derechos-humanos-en-argentina-informe2012/ Consulta: 11 de agosto de 2018

BARTOLOMÉ, M. C. (2013). Teoría de las Relaciones Internacionales: Un abordaje general a la Teoría de las Relaciones Internacionales, Facultad de Derecho y Ciencias Sociales, Universidad de 
Belgrano. Disponible en: http://repositorio.ub.edu.ar/handle/123456789/3505. Consulta: 13 de agosto de 2018

BIETTI, L. M. (2009). Entre la cognición política y la cognición social: el discurso de la memoria colectiva en Argentina, en Discurso y Sociedad, ISSN 1887-4606, 3(1), pp. 44-89

BRAVO, N. (2012). h.i.j.o.s. en Argentina. La emergencia de prácticas y discursos en la lucha por la memoria, la verdad y la justicia, en Sociológica, 27 (76), mayo-agosto, pp. 231-248

BRYSK, A. (2005). “ ¿Global Good Samaritans?”: Human Rights Promotion as Foreign Policy-The Case of Sweden, GPACS Working Paper

BUSCONI, A.; GARCÍA, J. (2016). La Política Exterior Argentina de Derechos Humanos durante los gobiernos de Cristina Fernández y Mauricio Macri, en XII Congreso Nacional y V Congreso Internacional sobre Democracia, Facultad de Ciencia Política y Relaciones Internacionales, Mesa Redonda del Observatorio de Política Exterior Argentina

BUSSO, A. (2016). Los ejes de la acción externa de Cristina Fernández: ¿cambios hacia un nuevo horizonte o cambios para consolidar el rumbo? en Relaciones Internacionales, 50, pp.143-170. Disponible

en: https://rephip.unr.edu.ar/xmlui/bitstream/handle/2133/9139/Busso IRI N 50.pdf?sequence=3\&isAl lowed=y. Consulta: 13 de agosto de 2018

CALONGE COLE, S. (2006). La representación mediática: teoría y método, en Psic. da Ed., São Paulo, 23, pp. 75-102. Disponible en: http://pepsic.bvsalud.org/pdf/psie/n23/v23a05.pdf. Consulta: 13 de agosto de 2018

CASCOS BLANCOS (2014). Asistencia Humanitaria y Política Exterior Argentina: A una década del nuevo paradigma en la región latinoamericana y caribeña 2003-2013. Disponible en: http://www.cascosblancos.gov.ar/userfiles/libro_ashum_lectura_final_2da_edicin.pdf Consulta: 11 de agosto de 2018

CASLA SALAZAR, K. (2011). El papel de los Derechos Humanos en la política exterior de y en la Unión Europea: un modelo de análisis, en Relaciones Internacionales, 17. Disponible en: http://www.relacionesinternacionales.info/ojs/article/view/288.html. Consulta: 30 de septiembre de 2018

CASTILlO, C. I. (2015). Política Exterior Argentina y los medios de comunicación: La Cuestión Malvinas. Tesis doctoral del Programa de Gestión y Políticas de Comercio Internacional. Universidad Politécnica de Valencia.

CEBRELLI, A.; RODRÍGUEZ, M. G. (2013). ¿Puede (in)visibilizarse el subalterno? Algunas reflexiones sobre representaciones y medios, en $\operatorname{Tram}(p)$ as de la Comunicación y la cultura, Facultad de Periodismo y Comunicación Social, La Plata, en prensa

CORIGLIANO, F. (2011). Los derechos humanos en la política exterior argentina: de la restauración democrática al Bicentenario de la Revolución de Mayo (1983-2010), en SALTALAMACCHIA ZICCARDI, N.; COVARRUBIAS VELASCO, A., Derechos humanos en política exterior. Seis casos latinoamericanos, Miguel Ángel Porrúa, México, 268 pp.

CROXATTO, G. (2013). Democracia y derecho, Página/12, 26 de noviembre, Buenos Aires. Disponible en: https://www.pagina12.com.ar/diario/elpais/1-234381-2013-11-26.html. Consulta: 1 de agosto de 2018

D’AlOTTO, A. (2015). La Argentina en el Consejo de Derechos Humanos de la ONU, Página/12, 7 de octubre, Buenos Aires. Disponible en: https://www.pagina12.com.ar/diario/elpais/1-2832492015-10-07.html. Consulta: 3 de agosto de 2018

DE DIEGO, J. (2014). La prensa escrita durante el gobierno de Néstor Kirchner. Periodismo de opinión y disputas por el sentido político frente al proceso de construcción del kirchnerismo. Los casos de Clarín, La Nación y Página/12, Tesis doctoral, Doctorado en Ciencias Sociales, Facultad de Humanidades y Ciencias de la Educación, Universidad Nacional de La Plata. Disponible en: http://repositorio.una.edu.ar/handle/56777/1247. Consulta: 25 de julio de 2018 
DONNELLY, J. (1998). Human Rights: A New Standard of Civilization?, en International Affairs (Royal Institute of International Affairs 1944- ), 74 (1), pp. 1-23

FERNÁNDEZ, M. N. (2013). Mediatización de la política en la Argentina kirchnerista: figura presidencial, periodismo militante y disputas por la toma pública de la palabra, en X Jornadas de Sociología. Facultad de Ciencias Sociales, Universidad de Buenos Aires, Buenos Aires, 2013. Disponible en: http://cdsa.aacademica.org/000-038/631.pdf. Consulta: 25 de julio de 2018

GLANC, L. (2008). Memoria Activa y demandas de justicia en Argentina, en Revista IIDH, 47, pp. $101-125$

GIARDINELLI, M. (2013). Una carta para "La Nación”, Página/12, 13 de octubre, Buenos Aires. Disponible en: https://www.pagina12.com.ar/diario/elpais/1-231174-2013-10-13.html. Consulta: 1 de agosto de 2018

HERRERA FLORES, J. (2003). Los derechos humanos en el contexto de la globalización: tres precisiones conceptuales. Colóquio Internacional - Direito e Justiça no Século XXI. Coimbra, 29 a 31 de Maio. Disponible en: https://www.ces.uc.pt/direitoXXI/comunic/HerreraFlores.pdf. Consulta: 29 de septiembre de 2018

HORTA, J. (2013). Representaciones mediáticas. Tres notas sobre los procesos semióticos en los medios masivos, en Comunicación y Medios, 28, pp. 96-112

JOZAMI, E. (2015). Macri, derechos humanos y revanchismo, Página/12, 5 de enero, Buenos Aires. Disponible en: https://www.pagina12.com.ar/diario/elpais/1-263226-2015-01-05.html. Consulta: 02/08/2018.

KOZINER, N.; ZUNINO, E. (2013). La cobertura mediática de la estatización de YPF en la prensa argentina: un análisis comparativo entre los principales diarios del país, en Global Media Journal México, 10(19), pp. 1-25

MOREIRA, C.; BARBOSA, S. (2010). El kirchnerismo en Argentina: origen, apogeo y crisis, su construcción de poder y forma de gobernar, en Sociedade e Cultura, 13 (2), pp. 193-200. Disponible en: http://www.redalyc.org/pdf/703/70316920005.pdf. Consulta: 13 de agosto de 2018

PARSONS, W. (2007). Políticas públicas: una introducción a la teoría y a la práctica del análisis de políticas públicas, FLACSO, México DF

PAUSELLI, G. (2014). La política exterior argentina de derechos humanos. Foreign Affairs Latinoamérica. Disponible en: http://revistafal.com/la-politica-exterior-argentina-de-derechoshumanos/. Consulta: 13 de agosto de 2018

TORRES, V. (Sin Fecha). Aspectos discursivos de las narrativas de la memoria. El concepto de campo desde la perspectiva de Pierre Bourdieu en la construcción discursiva de la memoria colectiva en Argentina, en II seminario internacional Políticas de la Memoria, Centro Cultural de la Memoria Haroldo Conti

SAINTOUT, F. (2013). Medios y gobiernos populares en América Latina. Apuntes para una discusión, en Revista Argentina de Sociología, Consejo de Profesionales en Sociología, Buenos Aires, 9-10 (17-18), pp. 139-154

SOBRADOS LEÓN, M. (2011). La inmigración como problema. Percepciones sociales y representaciones mediáticas, en Prisma Social, 6, pp. 1-26

SOLÍS DELGADILLO, J. M. (2016). Los tiempos de la memoria en las agendas políticas de Argentina y Chile, Eudeba, Buenos Aires

ULANOVSKY, C. (1997). Paren las rotativas. Historia de los grandes diarios, revistas y periodistas argentinos $\left(1^{\circ} \mathrm{ed}\right.$.). Espasa Calpe, Buenos Aires

WAINFELD, M. (2015). El legado después de tantos años, Página/12, 11 de octubre de 2015, Buenos Aires. Disponible en: https://www.pagina12.com.ar/diario/elpais/1-283552-2015-1011.html. Consulta: 2 de agosto de 2018 
Cuadernos de Política Exterior Argentina (Nueva Época), 129, junio 2019, pp. 75-90

ISSN 0326-7806 (edición impresa) - ISSN 1852-7213 (edición en línea)

ZUNINO, E. (2016). La cobertura mediática del "conflicto campo - gobierno" de 2008 en la prensa gráfica argentina. Un estudio comparativo de las agendas informativas sobre la Resolución $N^{o}$ 125/08 de los diarios Clarín, La Nación y Página/12. Tesis doctoral. Universidad Nacional de Quilmes.

Disponible en: https://ridaa.unq.edu.ar/bitstream/handle/20.500.11807/840/TD_2016_zunino_009.pdf?sequence=1 \&isAllowed=y. Consulta: 13 de agosto de 2018 